\title{
Enhanced ion acoustic lines due to strong ion cyclotron wave fields
}

\author{
H. Bahcivan and R. Cosgrove \\ Center for Geospace Studies, SRI International, Menlo Park, CA, USA \\ Received: 7 March 2008 - Revised: 9 June 2008 - Accepted: 23 June 2008 - Published: 31 July 2008
}

\begin{abstract}
The Fast Auroral Snapshot Explorer (FAST) satellite detected intense and coherent 5-20 m electric field structures in the high-latitude topside auroral ionosphere between the altitudes of $350 \mathrm{~km}$ and $650 \mathrm{~km}$. These electric fields appear to belong to electrostatic ion cyclotron (EIC) waves in terms of their frequency and wavelengths. Numerical simulations of the response of an electron plasma to the parallel components of these fields show that the waves are likely to excite a wave-driven parallel ion acoustic (IA) instability, through the creation of a highly non-Maxwellian electron distribution function, which when combined with the (assumed) Maxwellian ion distribution function provides inverse Landau damping. Because the counter-streaming threshold for excitation of EIC waves is well below that for excitation of IA waves (assuming Maxwellian statistics) our results suggest a possible two step mechanism for destabilization of IA waves. Combining this simulation result with the observational fact that these EIC waves share a common phenomenology with the naturally enhanced IA lines (NEIALS) observed by incoherent scatter radars, especially that they both occur near field-aligned currents, leads to the proposition that this two-step mechanism is an alternative path to NEIALS.
\end{abstract}

Keywords. Ionosphere (Ionospheric irregularities; Plasma waves and instabilities) - Space plasma physics (Electrostatic structures)

\section{Introduction}

The large-aperture, large-power incoherent scatter radars (ISR) are designed specifically to detect thermal electron density fluctuations and therefore are extremely sensitive to plasma instabilities. The naturally enhanced ion acoustic

Correspondence to: $\mathrm{H}$. Bahcivan

(hasan.bahcivan@sri.com) lines (NEIALs) are the radar backscatter from ion acoustic (IA) waves that are significantly enhanced above the thermal fluctuation levels. These lines have been of considerable interest because they may be signatures of strong field-aligned currents and/or plasma turbulence in the auroral ionosphere. The purpose of this paper is to show that NEIALs can be associated with electrostatic ion cyclotron (EIC) wave-like intense and sporadic spiky turbulent electric fields in the auroral ionosphere.

A summary of NEIALs characteristics is as follows. The radar enhancements occur during intense red aurora and soft electron precipitation; the enhancements are usually $2-3$ orders of magnitude larger than the quiescent values (such large enhancements cannot be associated with field-aligned irregularities because the relevant ISR observations were made with the radar beam directed far away from perpendicularity to the magnetic field); the enhancements occur at a range of altitudes above $250 \mathrm{~km}$, mostly occurring near $500 \mathrm{~km}$, and can reach $1500 \mathrm{~km}$; radar interferometric observations show that their transverse extent is several hundred meters; they last less than a few tenths of a second; the upshifted and downshifted spectral lines are not equally enhanced; the enhancements can more frequently be seen at $224 \mathrm{MHz}$ as opposed to at $930 \mathrm{MHz}$ and $1290 \mathrm{MHz}$ (Collis et al., 1991; Wahlund et al., 1993; Rietveld et al., 1996; Cabrit et al., 1996; Forme et al., 2001; Grydeland et al., 2004). For an extensive review of NEALS, including observations and theories, see Sedgemore-Schulthess and St.-Maurice (2001).

IA waves can be excited because of instabilities of twostream type, most likely the current driven electrostatic ion acoustic (CDEIA) instability and ion-ion two stream instability (St-Maurice et al., 2007; Collis et al., 1991; Foster et al., 1988; Wahlund et al., 1992). Because the radar enhancements occur during soft electron precipitation conditions, a first thought is that the soft electrons are stopped by the neutral atmosphere and the current carried by the precipitating electrons closes into the thermal electrons, which

Published by Copernicus Publications on behalf of the European Geosciences Union. 
resonantly interact with the IA oscillations. However, one problem with this mechanism is that current densities of more than $1 \mathrm{~mA} / \mathrm{m}^{2}$ are required to trigger the CDEIA instability in the upper ionosphere. This current density is in excess of typically measured field-aligned currents, although there are recent theoretical and experimental studies that show that the threshold for the CDEIA instability is significantly reduced if there is a transverse gradient in the relative magnetic field aligned drift (Gavrishchaka et al., 1998) or if the electron plasma is heated by Langmuir turbulence that leads to a higher $T_{e} / T_{i}$ ratio (Mishin and Fiala, 1995). Moreover, CDEIA instability cannot explain simultaneous enhancements of both ion lines - a turbulent excitation process may explain the simultaneous enhancements. Forme et al. (2001); Kontar and Pécseli (2005) suggested that simultaneous enhancements of both ion lines can occur through parametric excitation of IA waves by coupling to beamdriven Langmuir waves. The simultaneous radar observation of enhanced Langmuir waves and IA waves by Stromme et al. (2005) supports the parametric excitation mechanism, however, it is not a proof because the enhancements could be occurring independently.

The goal of this paper is to contribute to these studies by showing that IA oscillations are also sensitive to a different kind of energy source. We are going to consider EIC wavedriven IA excitations because: (1) there is in-situ evidence for strong EIC waves in the regions of enhanced backscatter observations, (2) the counter streaming current threshold for excitation of EIC waves is well below that for IA waves when the statistics are assumed to be Maxwellian, and (3) previous kinetic simulations, e.g. Rowland and Palmadesso (1981), showed that strong EIC wave fields can potentially distort the electron distribution function (EDF) $f(x, v, t)$. The starting point of this work is that the observed EIC-like waves have already been initiated by a field-aligned current instability mechanism, perhaps aided by shears and temperature anisotropy, and we want to determine if IA waves can be excited by such EIC waves. We will carry out kinetic simulations for ion wave amplitudes comparable to the FAST satellite measurements and from the simulation we will compute the incoherent scatter spectra. To the extent that the spectrum is computable (the backscatter can be analyzed in terms of the fluctuation-dissipation theorem), the peak-to-valley ratio of the spectrum will be indicative of a near-instability condition.

In the next sections, we are going to (1) present the FAST electric field measurements and discuss their EIC-like features, (2) describe a kinetic (Vlasov) code for computing the electron plasma response to ion wave fields and compute the electron distribution function, and (3) perform a Nyquist stability analysis and calculate the incoherent scatter spectrum analysis from the computed distributions.

\section{Electrostatic ion cyclotron waves}

EIC waves are considered important plasma wave modes in and near auroral arcs. Field-aligned currents are considered as the primary energy source for these waves. Some of the field-aligned currents observed by spacecraft and groundbased instrumentation are sufficiently strong to excite the current-driven ion cyclotron instability (Kindel and Kennel, 1971; Satyanarayana et al., 1985; Ganguli et al., 1994).

Some examples of FAST spacecraft electric field measurements from $360 \mathrm{~km}$ altitude are shown on the left side of Fig. 1 between 4.16 and $4.19 \mathrm{~s}$, and between 4.21 and $4.22 \mathrm{~s}$ past 04:36 UT. The top and bottom panels show the time series of electric field measurements made by DC coupled electric field antenna of lengths, 5 and $56 \mathrm{~m}$, respectively. The oscillations display a nearly sinusoidal wave form implying a spatially pure source. Under the "frozen-in" structures assumption, which is demonstrated to be valid below, the shortest scales are several meters in the direction transverse to the magnetic field. The right panels of Fig. 1 show their occurrence in a broader context. The V1-2 signal (top panel) and its power spectra (middle panel) show broadband ELF emissions (reaching above $1 \mathrm{KHz}$ ) near the boundaries of electron precipitation regions. The emissions are also in spatial proximity to large scale $(>100 \mathrm{~m}$ ) electric field structures superimposed on the 5-s period spin modulation. The EIC-like oscillations may be identified from the electric field power spectrum (middle panel) as broadband ELF spectral features above $500 \mathrm{~Hz}$. However, because of their extremely short durations $(\approx 0.05 \mathrm{~s})$, they are more easily distinguished from the electric field time series as isolated, spiky, and oscillatory events as shown in the left panels of Fig. 1.

Another example of measurements from a higher altitude $(562 \mathrm{~km})$ is shown in Fig. 2 and demonstrates a distinction of the subject small-scale electrostatic structures from electromagnetic oscillations with similar scales. The bottom panels are two subsets from the top panel, one coinciding with the intense DC magnetic perturbations and soft $(<1 \mathrm{keV})$ electron precipitation (on the left) and the other one occurring with no DC magnetic perturbations and in a region of hard $(>1 \mathrm{keV})$ electron precipitation (on the right). The bottomright panel shows a clear correlation between electric and magnetic fields indicating the electromagnetic nature of the oscillations, while the bottom-left panel shows no such correlation.

The Fourier transform of the FAST electric field measurement time series along the satellite track can be used as the kspectrum. This is so because the satellite velocity of greater than $8000 \mathrm{~m} / \mathrm{s}$ far exceeds the electrostatic turbulence phase velocities (perpendicular to B) of several $100 \mathrm{~m} / \mathrm{s}$, hence the observed phase structuring is due to the spacecraft traversing spatial structures. We can demonstrate this with sample multi-point electric field measurements. For example, the left side of Fig. 3 shows the approximate configuration of all the FAST electric field antennas and the right side shows the 

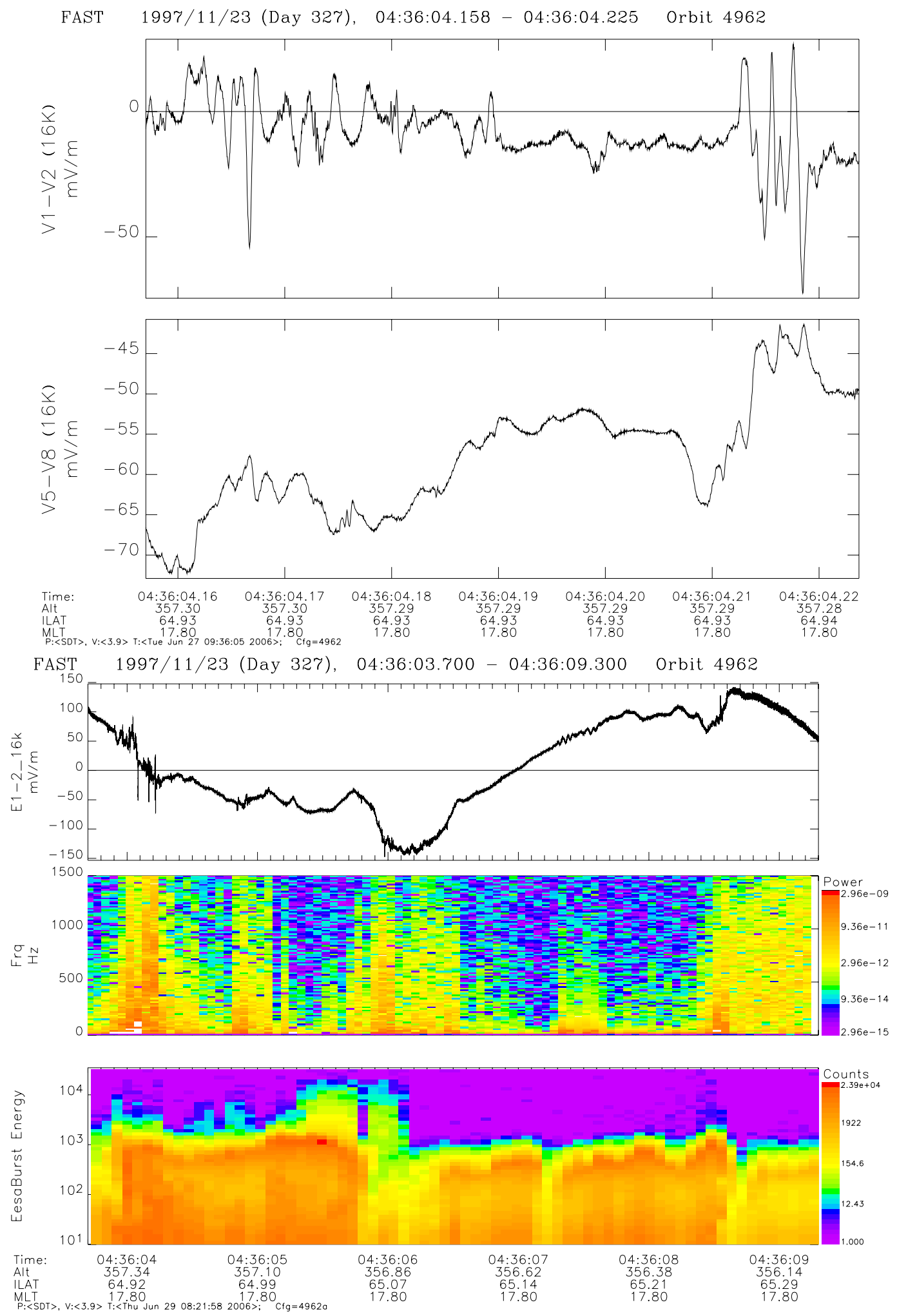

Fig. 1. Top two panels: FAST observations of EIC-like electric fields. The upper panel shows the V1-2 signal collected with a $5 \mathrm{~m}$ boom with a $2.5 \mathrm{~m}$ projection transverse to the magnetic field lines. The lower panel shows the V5-8 signal collected with a $56 \mathrm{~m}$ boom with a $48 \mathrm{~m}$ transverse projection. The booms are on the spin plane and the spacecraft is in a cartwheel configuration. Bottom three panels: the oscillations in a broader context, V1-2 signal (upper panel), electric field power spectrum (middle panel), and electron energy spectra (in eV) (lowest panel). 


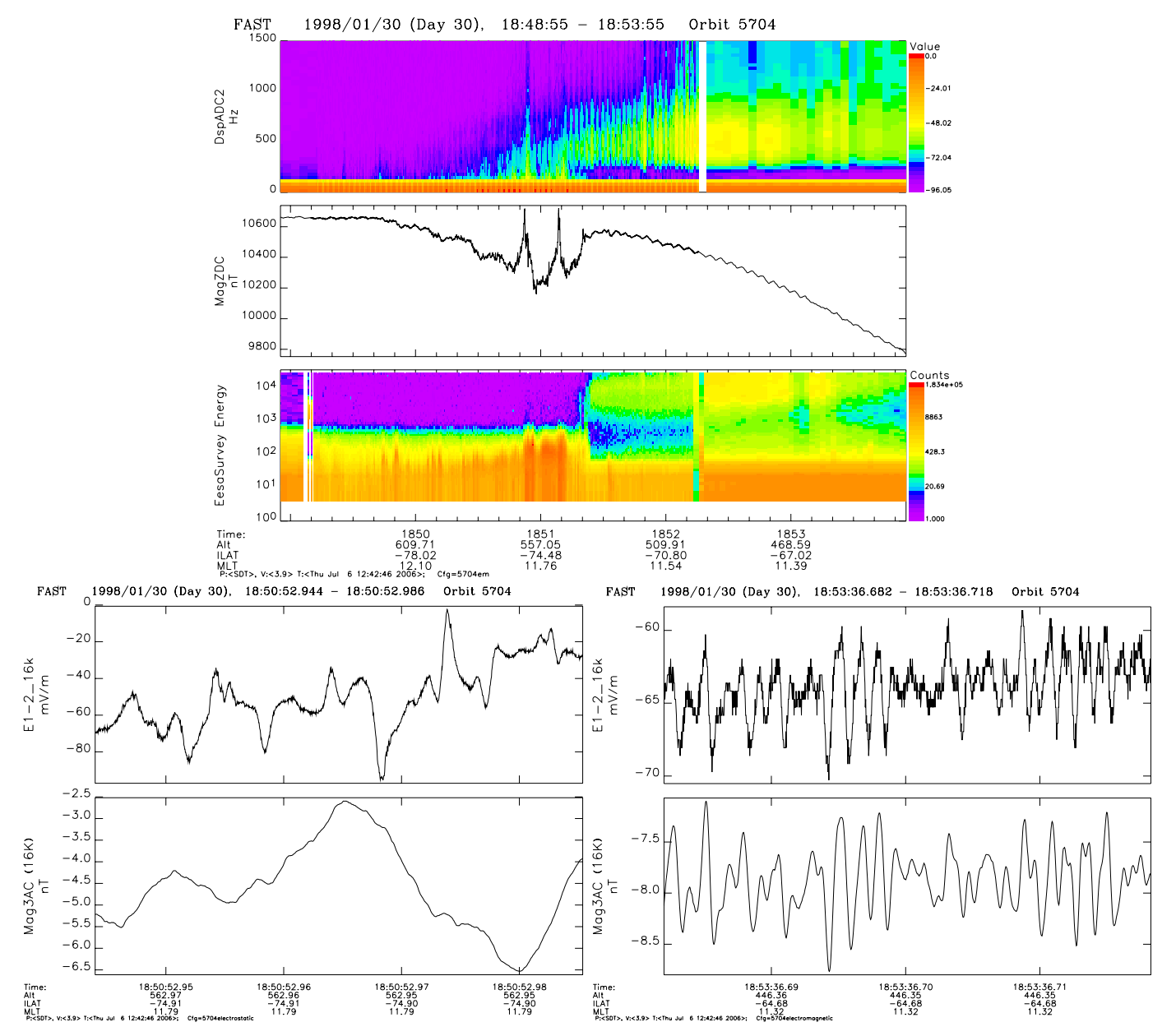

Fig. 2. The center-top panels show the electric field power spectrum, DC magnetic field perturbations along the spin axis, and electron energy spectra (in eV). The left-bottom panels show the V1-2 signal and the AC spin axis magnetic field perturbations from $0.04 \mathrm{~s}$ interval. The time of these left-bottom panels corresponds to the location of one of the two intense DC magnetic field perturbations shown in the center-top panels. The bottom-right panel shows the same signals as the bottom-left panels show, except that the time interval corresponds to a location in the hard $(>1 \mathrm{keV})$ electron precipitation period of the center-top panels and away from DC magnetic field perturbations.

time series of electric field measurements given by the potential differences between spheres 1 and 2, 5 and 6 , and 5 and 8 , all of which are located on the spin plane. Clearly, the V1-2 signal is very similar (after sign reversal) to the V5-6 signal but with a lag $(\approx 4 \mathrm{~ms})$ corresponding to the separation of the antennas $(\approx 70 \mathrm{~m})$ divided by the satellite speed $(\approx 8 \mathrm{~km} / \mathrm{s})$. This reappearance of the V5-6 signal wave form in the V1-2 signal after a time delay that matches the time it takes for the spacecraft to traverse their separation clearly supports the "frozen-in" assumption. However, it also possible that the waveforms have periods $10 \mathrm{~ms}$ or longer (at least several times longer than $4 \mathrm{~ms}$ ). This means the frequency is $<100 \mathrm{~Hz}$, which would include the $\mathrm{O}^{+}$gyrofrequency of $\approx 40 \mathrm{~Hz}$. Furthermore, the V5-8 signal is smoother, a consequence of nulling that can be expected as the wavelengths approach the dimensions of the electric field dipoles (LaBelle and Kintner, 1989). Because the V5-6 signal has similar waveform as the V1-2 signal and the nulling effect is observed in the V5-8 signal indicate that the oscillations should have spatial scales between several meters and a few decameters.

The electrostatic structures in the above examples are interpreted as EIC oscillations for several reasons. First, their coincidence with strong DC magnetic perturbations suggests their association with field-aligned currents, a primary free energy source to excite the current-driven EIC instability. Second, their scale lengths approximately matches the wavelength at which EIC instability growth maximizes. For instance, the oscillations shown in Fig. 1 were detected at $360 \mathrm{~km}$ altitude, which falls in a collisional region for the EIC instability (Satyanarayana et al., 1985). The predicted EIC instability growth rate maximizes for $k_{\perp} \rho_{i} \geq 1$, 

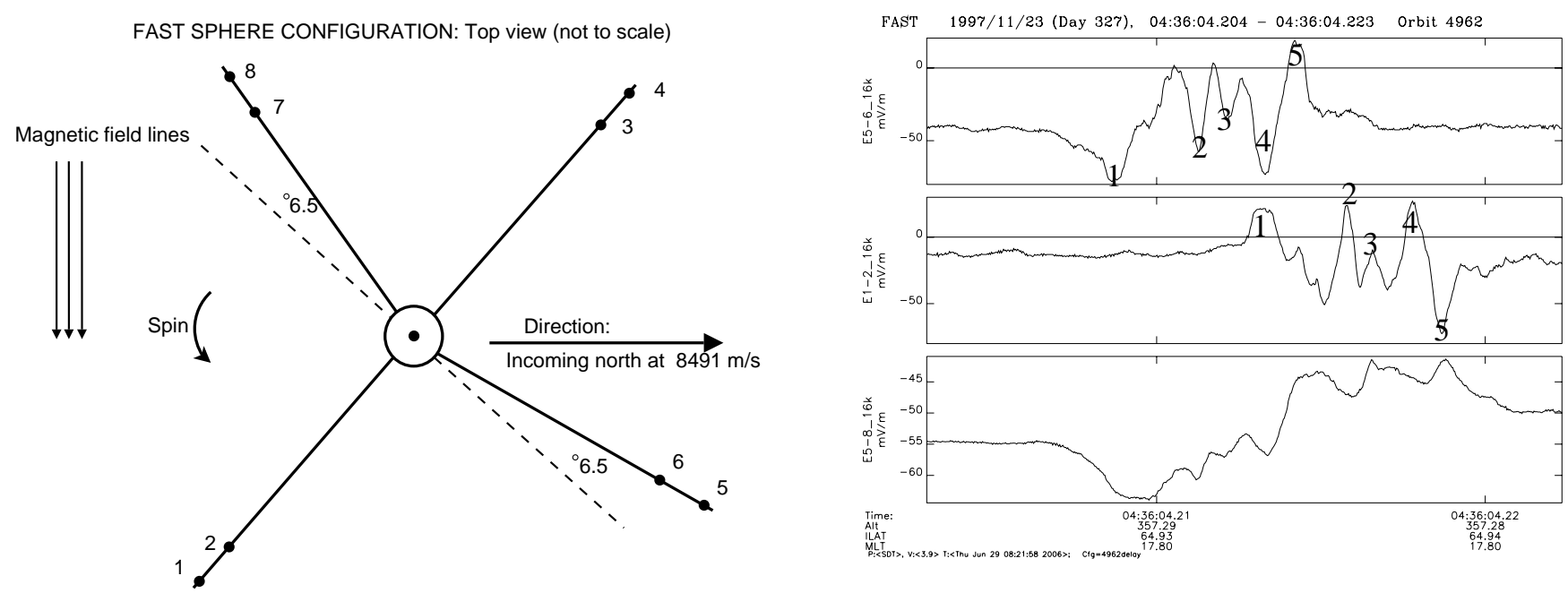

Fig. 3. Left panel: A schematic view of the electric field sensors on the FAST satellite at $4.21 \mathrm{~s}$ past 04:36 UT. The EIC-like oscillations of this time are seen on the right panel. Right panel: High-resolution electric field waveforms of the sensors V5-6, V1-2, and V5-8. The numbering 1 to 5 identifies spikes that can be matched (after sign reversal) from V5-6 signal to V1-2 after a time delay of approximately $4 \mathrm{~ms}$.

where $\rho_{i}$ is the ion gyroradius and $k_{\perp}$ is the wavenumber component transverse to the magnetic field lines. For an ion temperature of $1300^{\circ} \mathrm{K}$ and $B_{0}=4.2 \times 10^{-5} \mathrm{~T}$ we have $k_{\perp}=0.311 / m=2 \pi / \lambda$, and the instability maximizes for $\lambda \leq 20.47 \mathrm{~m}$. The oscillations seen in Fig. 1 have scales from several meters to a few decameters, in agreement with the scales predicted by the linear theory. The third reason is that the observed wave amplitudes match the predicted broadband electric field amplitudes when the turbulence saturates by ion resonance broadening (Palmadesso et al., 1974):

$\frac{(\delta E)^{2}}{8 \pi}=\frac{k_{\perp}^{2}}{2 \pi e^{2}} T_{i}^{2}\left(\frac{\omega-\Omega_{i}}{\Omega_{i}}\right)^{2}$

Considering the mode with the maximum linear growth rate (Satyanarayana et al., 1985), we have $k_{\perp}^{2} \rho_{i}^{2}=1$ and $\omega=1.2 \Omega_{i}$, and for $T_{i}=1300 \mathrm{~K}$ the resulting wave electric field amplitude is $16 \mathrm{mV} / \mathrm{m}$. The oscillations we observe are around tens of $\mathrm{mV} / \mathrm{m}$ range and therefore in satisfactory agreement with the theory.

Previous studies using an order lower velocity spacecraft, i.e. sounding rockets, detected similar structures at similar altitudes and similar proximities to field-aligned currents and suggested that the structures are EIC waves driven by field aligned currents. Bering et al. (1975) detected narrowband electric field oscillations with a sounding rocket at approximately $350 \mathrm{~km}$ altitude, in a region where the differential drift between ions and electrons was sufficient to destabilize the EIC wave. The detected wave forms are similar to the FAST measurements shown in Fig. 1 in terms of magnitude and scale. The rocket measurements were assumed to be EIC waves because of the narrow bandwidth of the oscillations and because their frequency range, after adjusting for the Doppler shift due to the rocket velocity, was in agreement with this mode. Later, Bering (1984) detected similar waves with a different rocket in diffuse aurora at lower altitudes $(\approx 150-250 \mathrm{~km})$. There were narrow peaks in the close proximity of the $\mathrm{O}^{+}$and $\mathrm{H}^{+}$cyclotron frequencies and EIC waves were suggested to be the possible causes for these oscillations. More recently, Bonnell et al. (1996) using plasma wave interferometry reported the detection of $\mathrm{H}^{+}$EIC waves at $\approx 850 \mathrm{~km}$ altitude by AMICIST (interferometry was possible because spacecraft velocity was much smaller than $\mathrm{H}^{+}$ cyclotron wave parallel or perpendicular phase velocity).

At somewhat higher altitudes in the topside auroral ionosphere the Freja spacecraft $(1700 \mathrm{~km})$ detected BB-ELF emissions containing a mixture of Alfvén, ion cyclotron, as well as IA waves (Wahlund et al., 1998). The Astrid-2 $(1000 \mathrm{~km})($ Holback et al., 2001) detected similar BB-ELF emissions.

The electrostatic turbulence we consider here must be distinguished from similar scale turbulence, some of which containing electromagnetic components. Satellites commonly detect small scale Alfvénic structure in the auroral region with energetic ion gyroradii scale sizes (Louarn et al., 1994; Mozer et al., 1997; Ergun et al., 1998; Cattell et al., 1998; Stasiewicz et al., 2000). Moreover, the $\boldsymbol{E} \times \boldsymbol{B}$ (gradient drift) interchange instability leads to an irregularity spectrum with a power-law form that extends in scale from $10 \mathrm{~km}$ down to the ion gyroradius (Tsunoda, 1988). By contrast, the oscillations we see are very localized, quasi-periodic, and spiky events. Their spectrum is often narrowband with no resemblance to a power-law form. Furthermore, a toroidal distribution of ion velocity transverse to the magnetic field lines can induce the Post-Rosenbluth instability at $0.5-1 \mathrm{~m}$ 
Table 1. Numerical simulation parameters.

\begin{tabular}{lcc}
\hline Parameter & value & units \\
\hline EIC wave $\lambda_{\|}$ & 256 & $\mathrm{~m}$ \\
EIC wave "linear" growth rate $(\gamma)$ & 10 & $1 / \mathrm{s}$ \\
Electron temperature & 3000 & $\mathrm{~K}$ \\
Electron thermal velocity & 213 & $\mathrm{~km} / \mathrm{s}$ \\
Number of spatial grids & 256 & \\
Simulation size & 256 & $\mathrm{~m}$ \\
Ion temperature & 1000 & $\mathrm{~K}$ \\
IA wavelength & 11.60 & $\mathrm{~cm}$ \\
Velocity interval for velocity distribution & 4 & $\mathrm{~km} / \mathrm{s}$ \\
Velocity interval after interpolation & 2 & $\mathrm{~m} / \mathrm{s}$ \\
Time step & 2 & $\mu \mathrm{s}$ \\
Electron collision frequency $v_{e}$ & 1000 & $1 / \mathrm{s}$ \\
\hline
\end{tabular}

wavelengths (Ott and Farley, 1975). This instability cannot be a cause for the oscillations because its frequency is of the order of the lower hybrid frequency, which is outside the possible frequency range we infer. For this same reason, the oscillations should also be distinguished from upper hybrid wave packets (LaBelle et al., 1989) of similar scale sizes.

\section{Electron distribution function distorted by EIC local wave fields, stability of IA waves, and enhanced inco- herent scatter radar spectra}

The purpose of this section is to show that electron distribution function (EDF) perturbations, similar to those in the simulation works by Rowland and Palmadesso (1981), imply IA wave growth and therefore ISR spectral enhancements.

\subsection{Electron distribution function}

The analysis consists of setting an ion wave as the parallel component of a small aspect angle EIC wave and computing the motion of a column of electron plasma by solving a kinetic equation in one dimension with the BGK collision term (Bhatnagar et al., 1954),

$$
\frac{\partial f}{\partial t}+v \frac{\partial f}{\partial x}+\frac{e \partial \phi / \partial x}{m_{e}} \frac{\partial f}{\partial v}=-v_{e}\left(f-f_{0}\right)
$$

We solve for the electric potential using Poisson's equation

$\epsilon_{0} \nabla^{2} \phi=n_{i}-n_{e}$

In Eq. (2), the background distribution function $f_{0}$ is uniform in space and Maxwellian in velocity, with the initial electron temperature. The electron density $n_{e}$ is obtained as

$n_{e}(x)=\int f(x, v) d v$
Starting with zero, the normalized ion wave amplitude is increased with a linear growth rate parameter $\gamma$, until $t=t^{\prime}$, as

$n_{i} / n_{0}=1+\left[\gamma\left(t-\left(t-t^{\prime}\right) \mathrm{u}\left(t-t^{\prime}\right)\right)\right] \sin \left(k_{\|} z+\omega_{\text {eic }} t\right)$,

where $\mathrm{u}$ is the unit step function. Two values of $t^{\prime}$ are chosen to achieve $n_{i} / n_{0}=0.1$ and $n_{i} / n_{0}=0.2$. The frequency of the ion wave is given by the EIC wave dispersion relation

$\omega_{\text {eic }}^{2}=\omega_{c}^{2}+k^{2} C_{s}^{2}$

where, $\omega_{c}$ is the ion cyclotron frequency, $k$ is the wavenumber, and $C_{s}$ is the ion sound velocity. The wavelength along the magnetic field is fixed at $\lambda_{\|}=256 \mathrm{~m}$, and the perpendicular wavelength is adjusted to give two different phase velocities along the magnetic field: $V_{p}=20 \mathrm{~km} / \mathrm{s}$, and $V_{p}=40 \mathrm{~km} / \mathrm{s}$. These choices result in $k_{\|} / k_{\perp} \approx 0.1$ and $k_{\perp} \approx 1 / \rho_{i}$, where $\rho_{i}$ is the ion gyroradious. In addition, for reference, we run the simulation with $V_{p}$ artificially set to zero.

Equations (2) and (3) are solved together using a finite difference method. Starting from the initial distribution function $f(x, 0)=f_{0}$, first the Poisson equation is solved and then $f(x, t)$ is iterated by $\Delta t$ in time using the fourth order Runge-Kutta method. Then, $n_{e}(x)$ is computed from $f(x, t+\Delta t)$, and $n_{i}$ is updated for the new time. The simulation is cycled this way until a stationary solution in the ion wave reference frame is reached, that is,

$\frac{\partial f\left(x+V_{p} t\right)}{\partial t}=0$.

Figure 4 shows the resulting EDF contour plots for wave density amplitudes of 20\% (left panels) and 10\% (right panels), and for $V_{p}=40,20$, and $0 \mathrm{~km} / \mathrm{s}$, top to bottom. Density maxima corresponds to flat topped EDFs, where low energy electrons have been trapped by potential wells (potential $\equiv$ electron potential energy). Density minima correspond to potential peaks and sharply peaked EDFs, where mid-to-high energy electrons have been depleted relative to low energy electrons. The depletion occurs because the mid to high energy electrons are constantly moving away, and must be replenished with electrons from the neighboring region. Upon imposition of the potential structure, the neighboring region (to the potential peak) is in a potential well, so that the electrons replenishing those with velocity $v$ must have a higher velocity $v+\Delta v$ at their origination point, that is, the replenishing electrons come from further down on the tail of the distribution function.

On the other hand, zero velocity electrons at the exact maxima of the potential structure never move away (in the case $V_{p}=0$ ), and hence need not be replenished. Their numbers are not decreased by imposition of the potential structure. These contrasting supply-and-demand scenarios for low energy, and for mid-to-high energy electrons leads to sharply peaked EDFs at the potential maxima. EDFs at the potential peaks (density minima) for $V_{p}=0$ are shown in Fig. $5 \mathrm{c}$, and $\mathrm{f}$. 
(a)
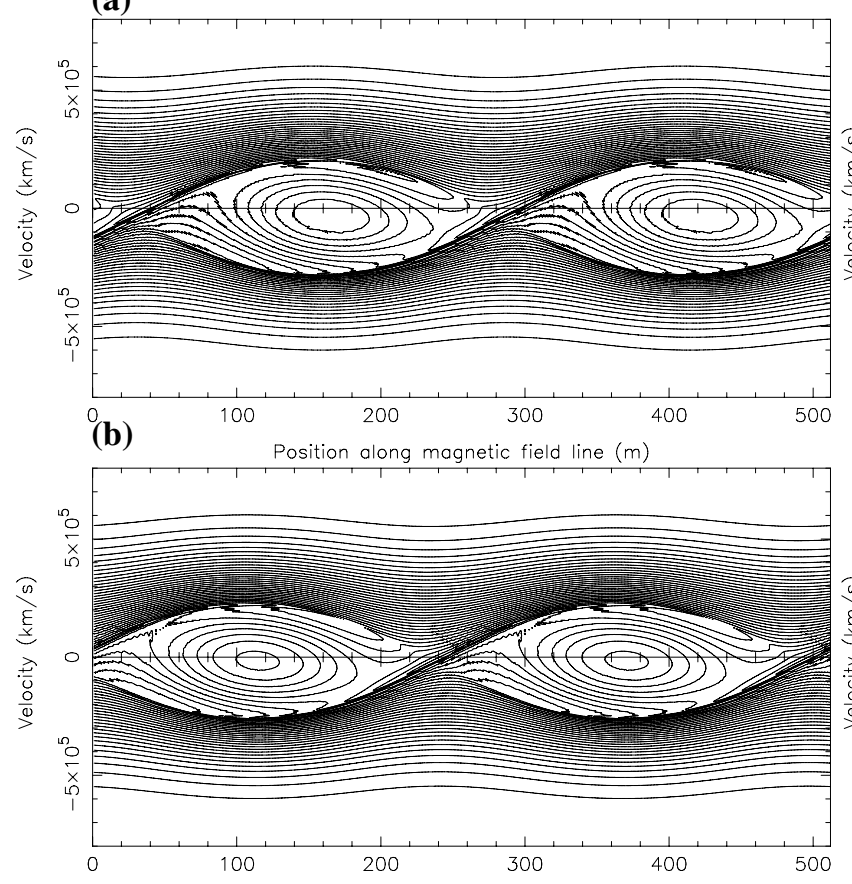

(c)

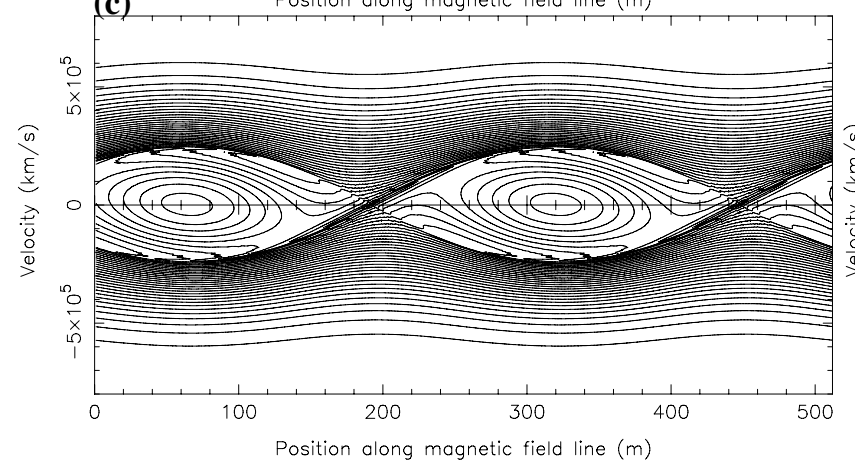

(d)

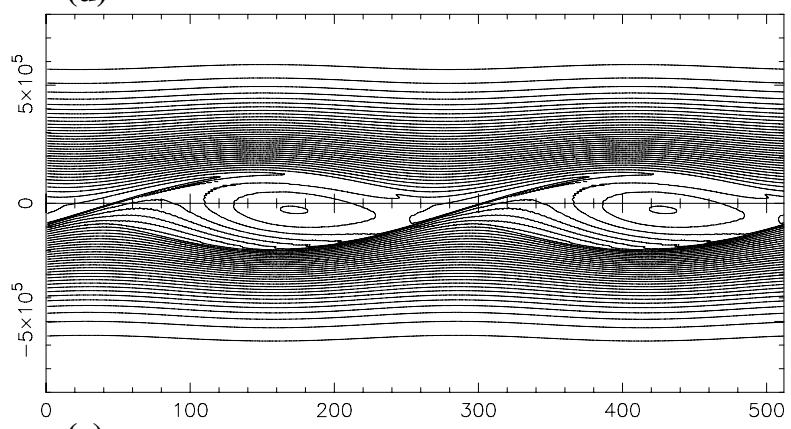

(e) Position along magnetic field line $(\mathrm{m})$
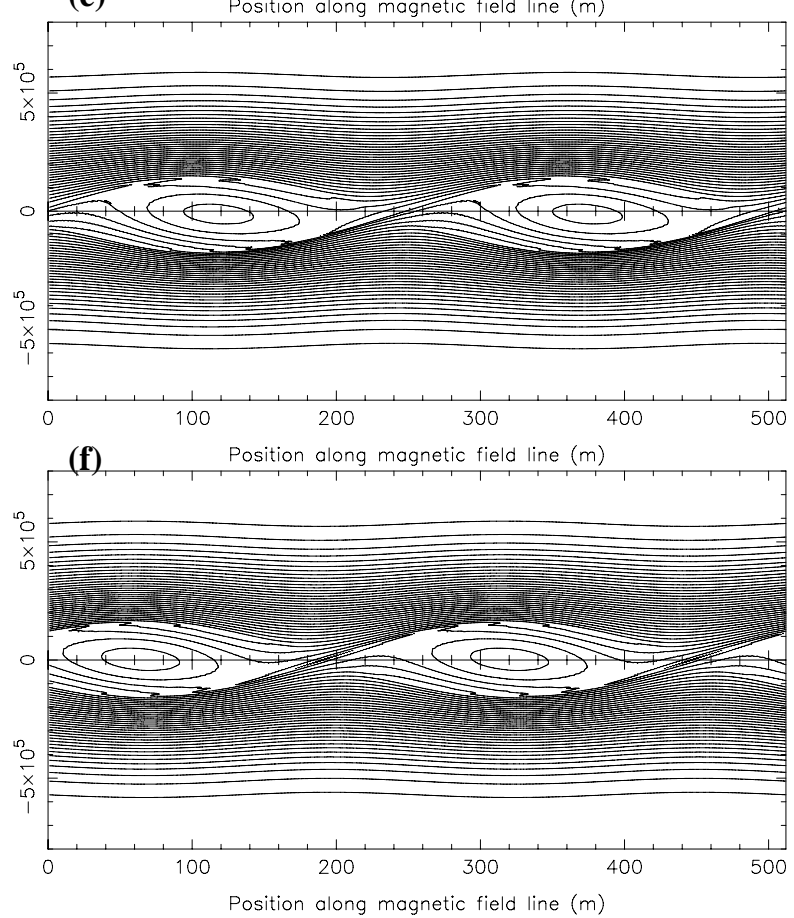

Fig. 4. Contours of EDF: (a) $n \equiv 20 \%$ and $V_{p}=40 \mathrm{~km} / \mathrm{s}$; (b) $n \equiv 20 \%$ and $V_{p}=20 \mathrm{~km} / \mathrm{s} ;$ (c) $n \equiv 20 \%$ and $V_{p}=0 \mathrm{~km} / \mathrm{s}$; (d) $n \equiv 10 \%$ and $V_{p}=40 \mathrm{~km} / \mathrm{s} ;$ (e) $n \equiv 10 \%$ and $V_{p}=20 \mathrm{~km} / \mathrm{s}$; (f) $n \equiv 10 \%$ and $V_{p}=0 \mathrm{~km} / \mathrm{s}$. The $256-\mathrm{m}$ long simulation box is repeated to $512-\mathrm{m}$.

In the case $V_{p}>0$ it is the electrons moving with the exact velocity $V_{p}$ that never move away from the potential maxima, and whose numbers experience the greatest increase according to the supply and demand scenario. Hence, the EDF maxima is shifted in the direction of the phase velocity of the EIC wave. The resulting EDFs, shown in Fig. 5a, b, d, and e, are similar to the case for $V_{p}=0$, except that they are not symmetrical, and have peaks shifted toward $V_{p}$.

3.2 Ion acoustic stability analysis and incoherent scatter spectrum

Destabilization of ion acoustic waves requires that the central peak of the EDF is shifted past the phase velocity of ion acoustic waves (about $1 \mathrm{~km} / \mathrm{s}$, defined with respect to the ion distribution function), such that the Landau damping from electrons becomes negative, and overcomes the (positive) Landau damping from ions. For a Maxwellian EDF the shift must be on the order of the electron thermal velocity, which implies a rather large electric field. However, for the sharply peaked EDFs shown in Fig. 5 the required shift is much smaller. We will analyze the stability of the EDFs obtained from our simulation using the Nyquist method.

The dispersion relation for electrostatic waves (e.g. Nicholson, 1983), which includes IA waves, is written

$$
\begin{aligned}
\epsilon & =0, \text { where } \\
\epsilon & =1+\epsilon_{i}+\epsilon_{e}=1+\frac{\omega_{e}^{2}}{k^{2}} \int_{C} \frac{\partial F / \partial u}{\omega / k-u} d u \\
F(u) & =F_{i}(u)+F_{e}(u)=\frac{m_{e}}{n_{0} m_{i}} \int f_{i} \delta(u-\hat{\boldsymbol{k}} \cdot \boldsymbol{v}) d \boldsymbol{v}+\frac{1}{n_{0}}
\end{aligned}
$$


(a)

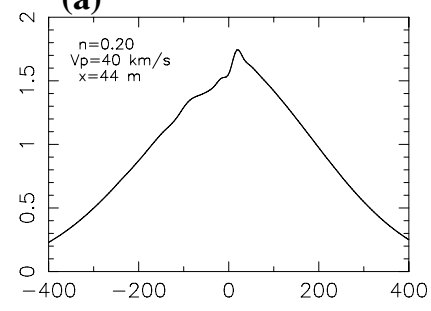

(b)

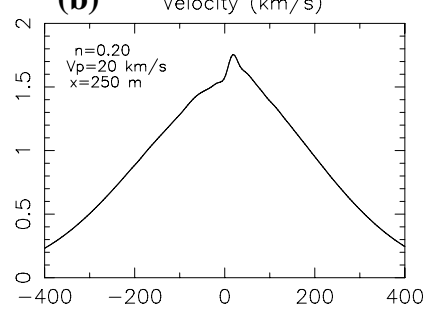

(c)

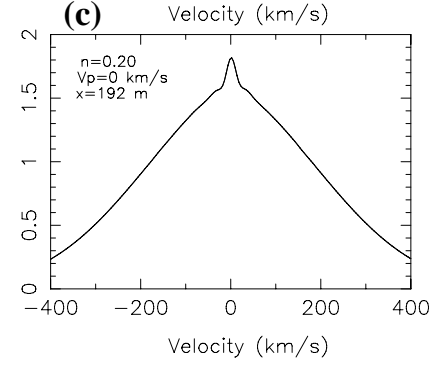

(d)

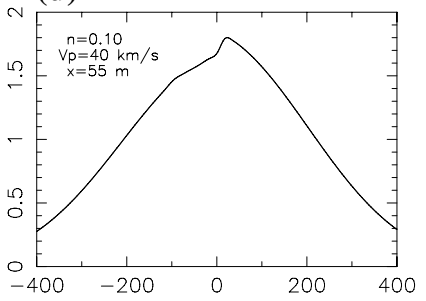

(e) Velocity $(\mathrm{km} / \mathrm{s})$

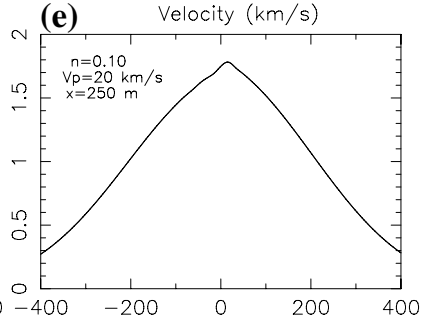

(f)

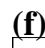

Velocity $(\mathrm{km} / \mathrm{s})$

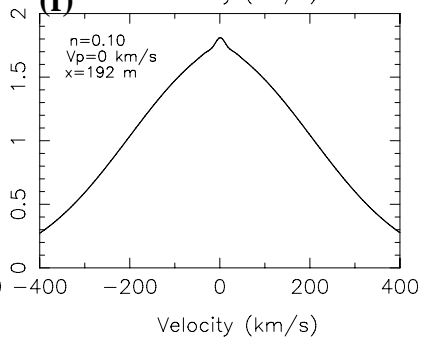

Fig. 5. Individual EDFs near density minimums.

$$
\int f_{e} \delta(u-\hat{\boldsymbol{k}} \cdot \boldsymbol{v}) d \boldsymbol{v},
$$

where $\hat{\boldsymbol{k}}$ is a unit vector along the wave vector, which in our case we take to be along the magnetic field. The system is unstable if the dispersion relation $\epsilon=0$ has solutions in the upper half of the complex frequency plane. The Nyquist method is to determine the number of such solutions by tracing out the path $\xi$ traversed by $\epsilon$ as $\omega$ encircles the upper half of the complex frequency plane (i.e. $-\infty<\omega<\infty$, since as $|\omega| \rightarrow \infty, \epsilon \rightarrow 1)$. As can be shown by a simple application of the residue theorem, the number of times that (the closed path) $\xi$ circles the origin in the counterclockwise direction is the number of zeros of $\epsilon$ in the upper half plane. (Circling in the clockwise direction is impossible.)

Figure 6 shows the curves $\xi$ obtained by using the EDFs shown in Fig. 5 in Eq. (7). Note that the curves $\xi$ are plotted for $-3<\omega / k<3 \mathrm{~km} / \mathrm{s}$ and they mostly correspond to the ion distribution function because the electron distribution function does not change much over this interval. Figure 7 shows a representative drawing of the curve $\xi$ for $-\infty<\omega / k<\infty$ where the dashed curve corresponds to the electron distribution function. The system is unstable for the case $V_{p}=40 \mathrm{~m} / \mathrm{s}$, $n_{i} / n_{0}=0.2$. Figure 9 shows $\xi$ at multiple positions for the same set of simulations as in Fig. 4. Note that there are 256 positions, and $\xi$ for every position is plotted. A plasma instability occurs if any of $\xi$ encircles the origin. The plasma

is stable for the stationary wave. In addition, for the same phase velocity, the increase of ion wave amplitude increases the number of curves encircling the origin.

The function $F$ is an effective distribution function determining the effects of ion and electron Landau damping on IA waves. Using the residue theorem, it can be shown that the imaginary part of the dispersion function $\epsilon$, for $\omega$ on the real axis, is $-\left.\pi \frac{\omega_{e}^{2}}{k^{2}} \frac{\mathrm{d} F}{\mathrm{~d} u}\right|_{u=\omega / k}$. Therefore, the curve $\xi$ can only cross the real axis when the derivative of $F$ vanishes. Because the imaginary part is positive for $\omega \rightarrow+\infty$, and negative for $\omega \rightarrow-\infty$, to circle the origin in the counterclockwise direction $\xi$ must cross the real axis at least twice. Therefore, a necessary (the Nyquist criteria is necessary and sufficient) criterion for instability is that the effective distribution function $F$ must have two humps. It is this fact that gives rise to the common wisdom that the EDF must shift sufficiently with respect to the ion distribution function for the system to become unstable. When the EDF is sharply peaked as in Fig. 5, the required shift is not as far, and this explains why the system has become unstable at a relatively modest electron drift. As a consistency check, a closeup of the function $F$ is shown in Fig. 10, showing the emergence of two humps.

Figure 8 (left panel) displays the EDFs at all 256 positions as a 3-D plot for the case $n \equiv 20 \%$ and $V_{p}=40 \mathrm{~km} / \mathrm{s}$. The right panel zooms into the first 64 positions and also shows the unstable positions with black curves. The dominant feature of this set of EDFs is the "ridge" that crosses from negative to positive velocities as we advance in position. The unstable curves occur near where the ridge crosses the zero velocity axis. This means that the sharp gradient of EDF in velocity associated with the ridge must occur near the zero velocity axis so that strong inverse electron Landau damping occurs. Although we have already smoothed the EDF's with a spline function, the EDFs are not entirely artifact free, which affects the IA stability. However, the "ridge" feature in the EDF is clearly not an artifact. Moreover, the clustering of the unstable cases near where the ridge crosses the zero velocity axis is evidence that the stability is determined by the ridge feature.

Figure 11 shows the mean electron drift along the magnetic field as a function of position along the wave. The peak electron drift for $20 \%$ wave amplitude is $\approx 10 \mathrm{~km} / \mathrm{s}$, which is much lower than the critical drift $\left(\approx v_{t e}\right)$ for IA instability required for Maxwellian electron and ions. This is a main point of this paper, that the current as inferred from the insitu magnetometers does not need to be near the critical drift for the IA instability to occur.

We find that the peak electric potential for $n_{i}=20 \%$ and $V_{p}=40 \mathrm{~km} / \mathrm{s}$, is $\approx 0.1 \mathrm{~V}$, which is higher than the Boltzman potential we estimate for the ion wave density. A flat-topped electron distribution and collisionality of the electrons results in a higher potential as the electron response to shield the ion wave is diminished by these factors. The peak electric potential corresponds to a peak perpendicular electric field of 

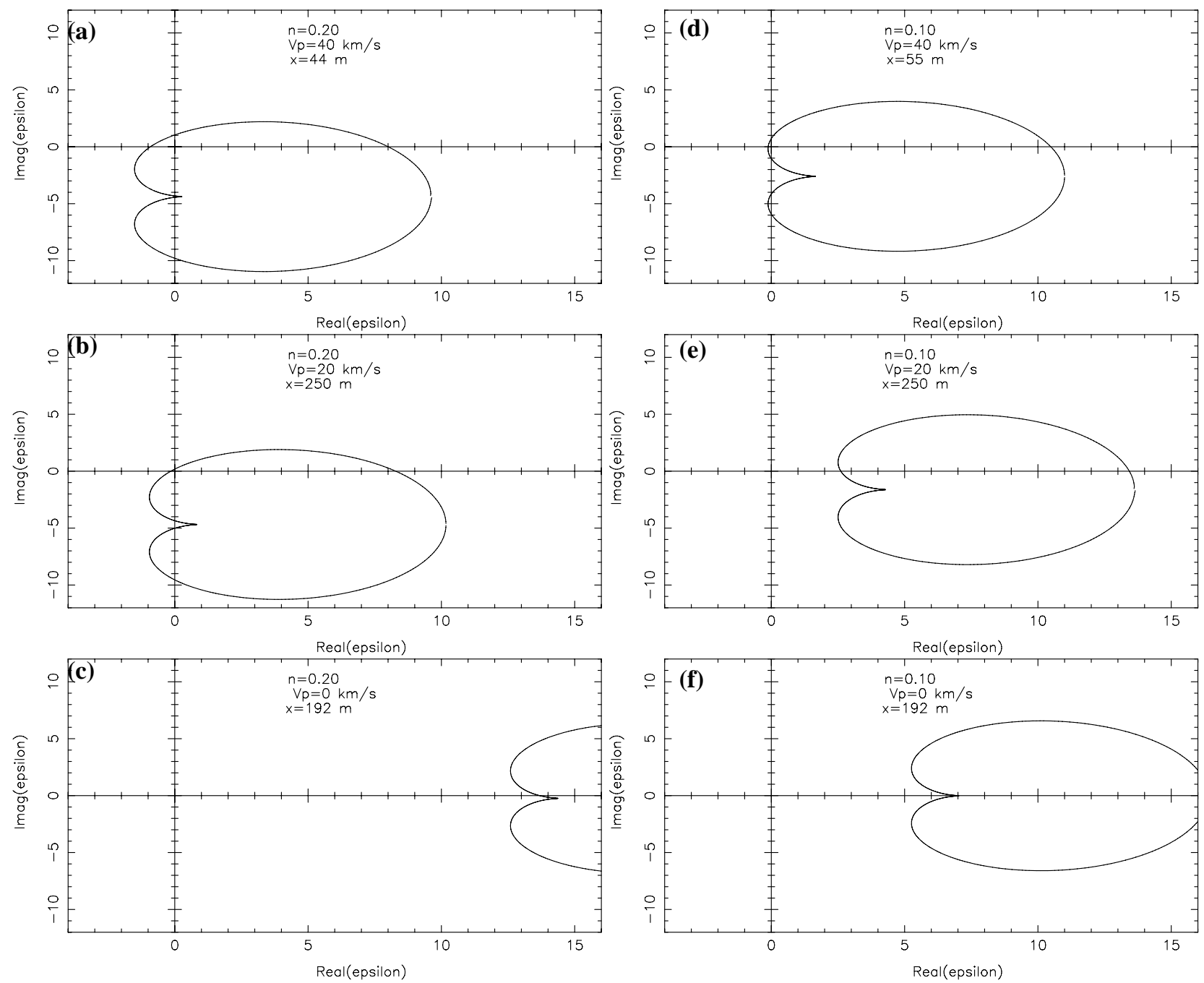

Fig. 6. The plasma dispersion curve $C_{\epsilon}$, found by mapping the curve $C_{\omega}$ for the EDFs shown in Fig. 5 .

$\approx 30 \mathrm{mV} / \mathrm{m}$ for $k_{\perp} \approx 1 / \rho_{i}$, which when multiplied by $1 / \sqrt{2}$, corresponds to an RMS $\delta E_{\perp}=21 \mathrm{mV} / \mathrm{m}$. This value is in good agreement with the predicted broadband electric field $(\delta E)$ of $16 \mathrm{mV} / \mathrm{m}$ calculated from Eq. (1), which is for turbulent saturation by ion resonance broadening (Palmadesso et al., 1974). In addition, the spiky electric fields measured by the FAST satellite, as seen in Fig. 1, have amplitudes of several $10 \mathrm{~s}$ of $\mathrm{mV} / \mathrm{m}$, sometimes reaching $100 \mathrm{mV} / \mathrm{m}$. We predict stronger EDF distortions and, therefore, a stronger IA instability for the measured electric fields.

The ISR spectrum can be calculated for the EDFs that are stable to IA waves. The ISR spectrum for a collisionless unmagnetized plasma with one Maxwellian ion species is given by (Dougherty and Farley, 1960):

$S(k, \omega)=\frac{1}{k}\left|\frac{1+\epsilon_{i}(\omega / k)}{\epsilon(\omega / k)}\right|^{2} F_{e}\left(\frac{\omega}{k}\right)$

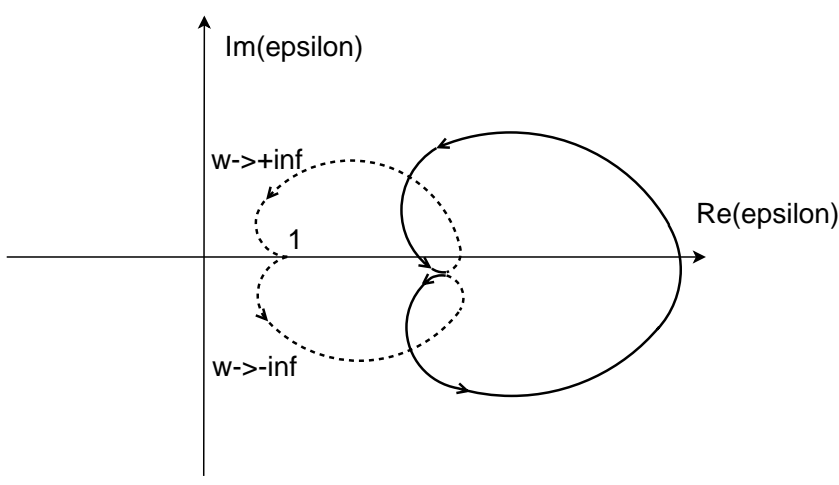

Fig. 7. Representative drawing of the plasma dispersion curve $C_{\epsilon}$ : ion $(|\omega / k|<3 \mathrm{~km} / \mathrm{s})$ (solid) and electron $(|\omega / k|>3 \mathrm{~km} / \mathrm{s})$ (dashed) features. The contour plots in Fig. 6 show only the ion feature. 

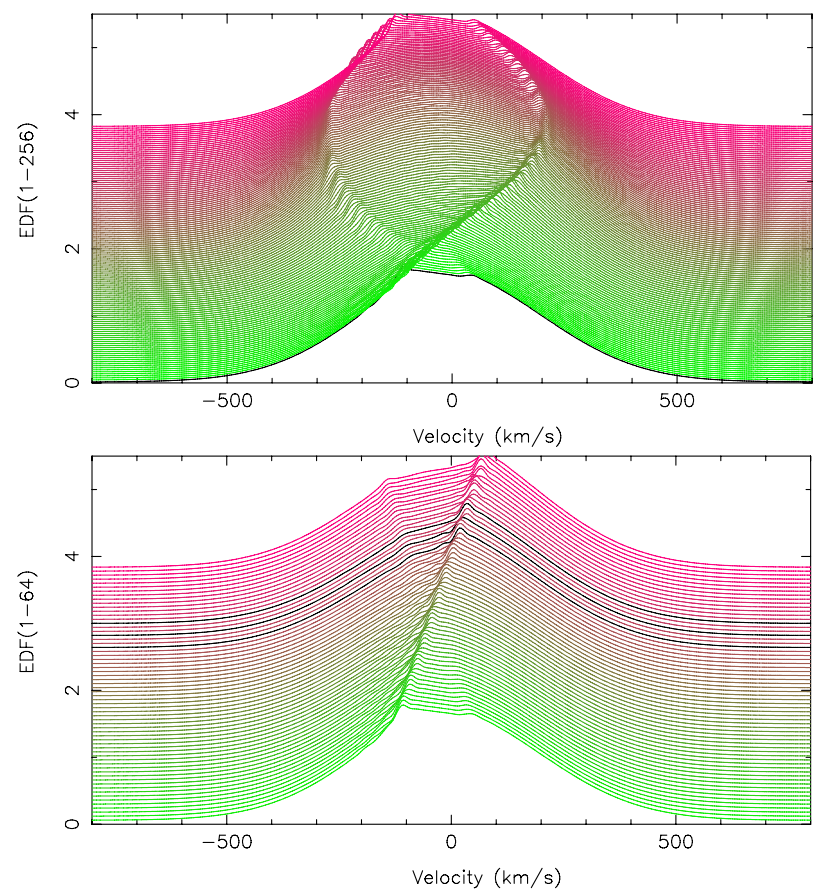

Fig. 8. Left: The EDF for $n \equiv 20 \%$ and $V_{p}=40 \mathrm{~km} / \mathrm{s}$ over all 256 positions. Right: The EDF over the first 64 positions; the lines in black are unstable.

$$
+\frac{m_{i} Z}{m_{e} k}\left|\frac{\epsilon_{e}(\omega / k)}{\epsilon(\omega / k)}\right|^{2} F_{i}\left(\frac{\omega}{k}\right),
$$

where $Z$ is the charge number of the ion species, $F_{e, i}$ are the one-dimensional velocity distributions along the radar lineof-sight, and $\epsilon$ is the plasma dispersion function, both of which are given in Eq. (7). The electron and ion characteristic frequencies are given as

$\omega_{0 e, i}=\left(\frac{n_{0} e^{2}}{\epsilon_{0} m_{e, i}}\right)^{1 / 2}$

The contour integral in Eq. (5) is evaluated as

$\int_{C}=P \int_{-\infty}^{\infty}-i \pi($ residue at $v=\omega / k)$

where the first term on the RHS is the principal value of the integral. For the ion species, we used a mass of 16 protons, corresponding to $\mathrm{O}^{+}$ions. The plasma can be considered collisionless for the purpose of ISR spectrum calculations because the (UHF/S-Band) ISR correlation time is much smaller than the mean time between collisions.

Figure 12 shows the ISR spectrum for $11.6 \mathrm{~cm}$ waves according to the Eq. (9) using the EDFs shown in Fig. 5. Note that the ISR calculations are inapplicable if the plasma is unstable; however, we may interpret a "spike" in the calculated shape as an indicator that the denominator in Eq. (9) is approaching zero, that is, a solution of the dispersion relation lies near the real $\omega$ axis. It is clear that those panels in Fig. 12 that contain spikes match to the panels of $C_{\epsilon}$ in Fig. 9 that indicate instability based on the Nyquist stability criteria.

\section{Discussion}

The numerical simulations here show that strong EIC-like wave fields lead to non-Maxwellian electron distribution functions that look like "phase-space holes", which are electric potential structures that trap some portion of the electron velocity distribution (see p. 89 in Paschmann et al., 2003, and the references therein). Previous auroral region studies associate the phase-space holes with the non-linear phase of plasma instabilities of mainly two-stream type, such as electron-ion drift and electron beams (e.g. Newman et al., 2001; Muschietti et al., 1999). In our case, phase-space holes occur periodically in space as a result of a large amplitude EIC wave potential structure along the magnetic field. The structure moves along the magnetic field with the parallel phase velocity of the EIC wave, i.e. $\omega_{\text {eic }} / k_{\|}$, which is much greater than the ion acoustic/thermal velocity. To determine whether these EIC wave-induced phase-space structures can be described in terms of an exact nonlinear electrostatic plasma structure, e.g. a Bernstein-Greene-Kruskal (BGK) electron hole (Bernstein et al., 1957), requires a separate analysis. The main interest here is, rather, on the transition region from one phase-space hole to another, and to determine if the structuring in the transition region near zero velocity could excite the IA instability.

The topic of wave-driven IA wave excitation was explored in our previous work (Bahcivan et al., 2006), which was on the enhancement of IA oscillations by the parallel electric fields of the Farley-Buneman waves in the $E$ region. In that work, we used a kinetic code with a detailed modeling of electron collisions and solved for the EDF for a spatially homogeneous plasma along the magnetic field for a given RMS local parallel electric field. The solution contained nonMaxwellian features that lead to enhancements of IA oscillations; however, an instability was unrealistic for a reasonable RMS parallel electric field in the collisional $E$ region. On the other hand, because the $F$ region electrons are much less collisional, the EDF could be easily distorted and an IA instability is more likely to occur.

The critical drift for the CDEIA instability is reduced if there is a transverse gradient in the relative magnetic field aligned drift. This effect was studied (Gavrishchaka et al., 1998, 2000), and experimentally demonstrated (Teodorescu et al., 2002; Agrimson et al., 2001). A transverse gradient is naturally present in an EIC wave structured electron flow because the electron drift is energized by EIC wave fields: in one phase the drift is upgoing, in another it is downgoing, and the opposing drifts are separated by $\lambda_{\perp} / 2$. The transverse gradient effect on the IA instability can be analyzed from the generalized dispersion relation developed by Ganguli et al. 

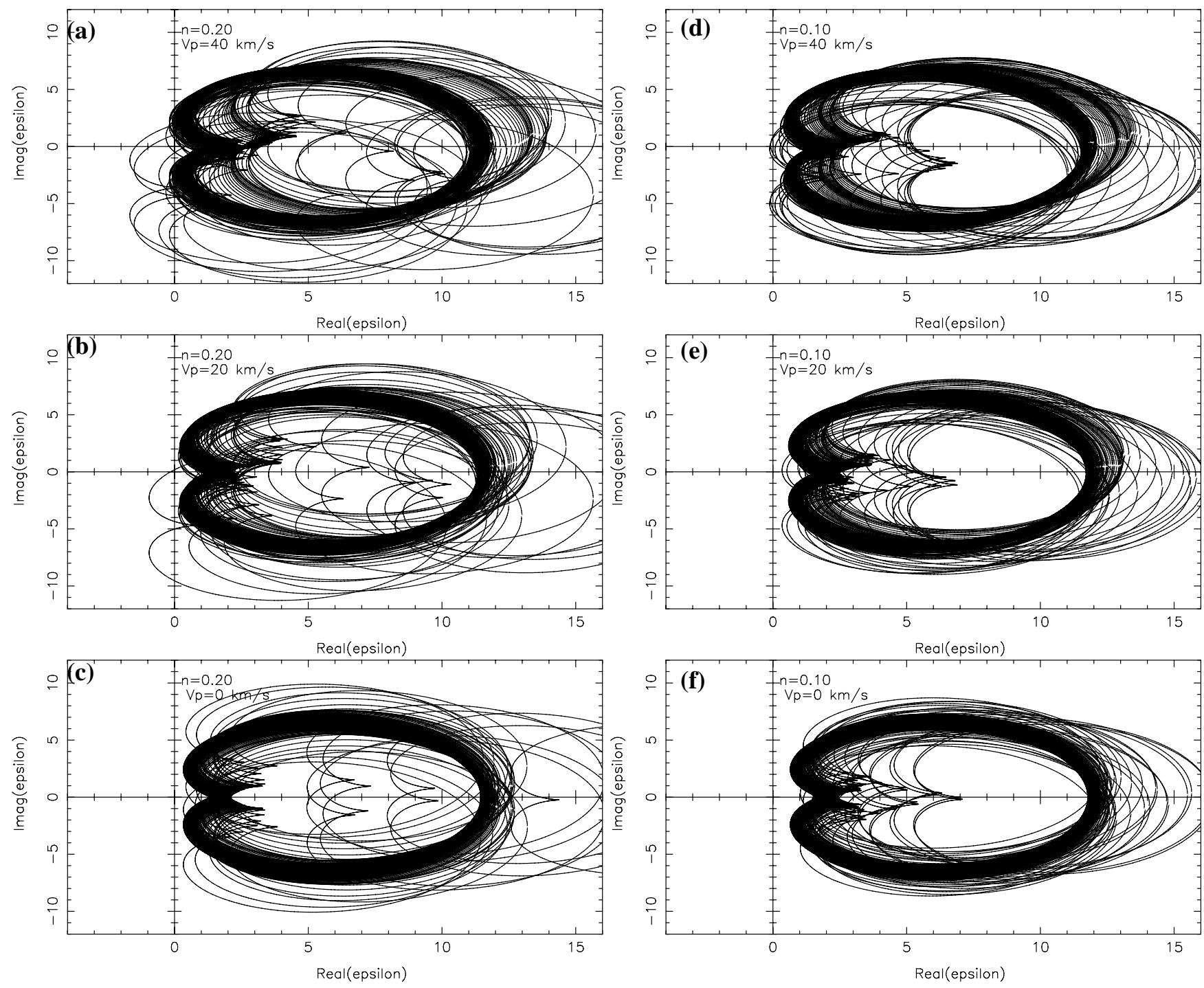

Fig. 9. The plasma dispersion curves $C_{\epsilon}$ plotted in each panel for all positions: (a) $n_{i} \equiv 20 \%$ and $V_{p}=40 \mathrm{~km} / \mathrm{s} ;$ (b) $n_{i} \equiv 20 \%$ and $V_{p}=20 \mathrm{~km} / \mathrm{s}$; (c) $n_{i} \equiv 20 \%$ and $V_{p}=0 \mathrm{~km} / \mathrm{s} ;$ (d) $n_{i} \equiv 10 \%$ and $V_{p}=40 \mathrm{~km} / \mathrm{s} ;$ (e) $n_{i} \equiv 10 \%$ and $V_{p}=20 \mathrm{~km} / \mathrm{s} ;$ (f) $n_{i} \equiv 10 \%$ and $V_{p}=0 \mathrm{~km} / \mathrm{s}$.

(1988). For Maxwellian ions and electrons, the generalized susceptibility functions $G_{e, i}^{\star}$ are related to the susceptibility functions in Eq. (5) as

$\epsilon_{e}^{\star}=\epsilon_{e}\left(1+\frac{V_{d e}^{\prime}}{\mu u \Omega_{i}}\right)$

$\epsilon_{i}^{\star}=\epsilon_{i}\left(1-\frac{V_{d i}^{\prime}}{u \Omega_{i}}\right)$

where $u \equiv k_{z} / k_{y}, V_{d \alpha}^{\prime}=d V(d \alpha) / d x$, and $\mu=m_{i} / m_{e}$. Here, $k_{y}$ and $k_{z}$ are the transverse and magnetic field aligned wavenumber components of the IA mode. The importance of the shear effect can be quantified as follows. Figure 4 shows the electron drift for the $20 \%$ wave amplitude: the drift is about $10 \mathrm{~km} / \mathrm{s}$. For simplicity assume that this drift represents that of Maxwellian electrons, although the simulation

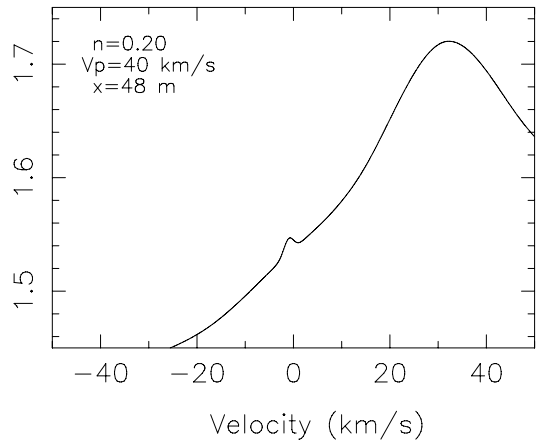

Fig. 10. The function $F$. 
(a)
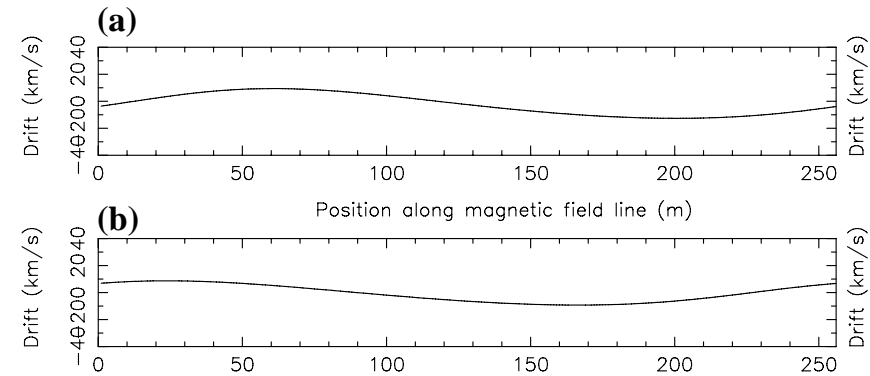

(c)

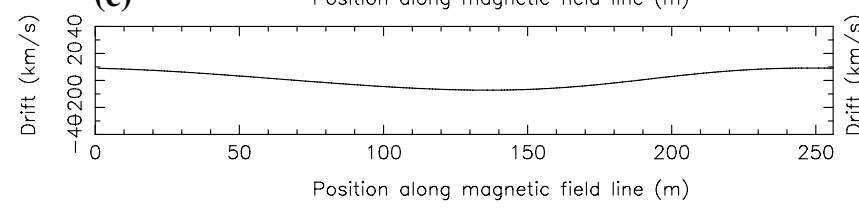

(d)

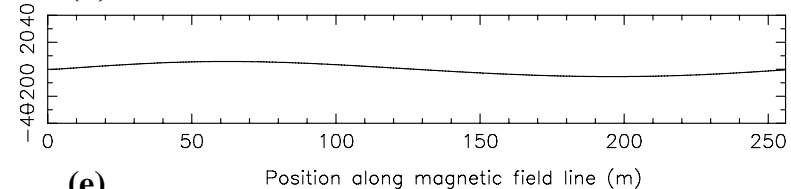

(e)

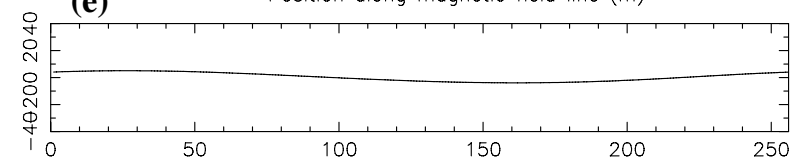

(f)

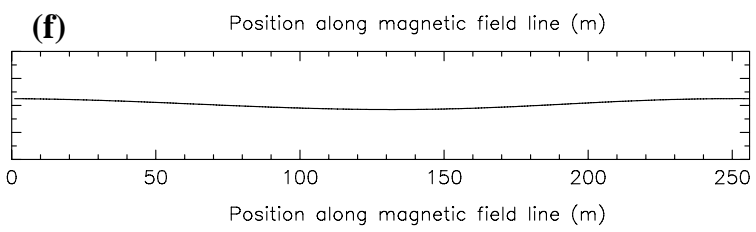

Fig. 11. The mean (parallel) electron drift as a function of position along the magnetic field: (a) $n \equiv 20 \%$ and $V_{p}=40 \mathrm{~km} / \mathrm{s}$; (b) $n \equiv 20 \%$ and $V_{p}=20 \mathrm{~km} / \mathrm{s} ;$ (c) $n \equiv 20 \%$ and $V_{p}=0 \mathrm{~km} / \mathrm{s} ;$ (d) $n \equiv 10 \%$ and $V_{p}=40 \mathrm{~km} / \mathrm{s} ;$ (e) $n \equiv 10 \%$ and $V_{p}=20 \mathrm{~km} / \mathrm{s} ;$ (f) $n \equiv 10 \%$ and $V_{p}=0 \mathrm{~km} / \mathrm{s}$.

(a)

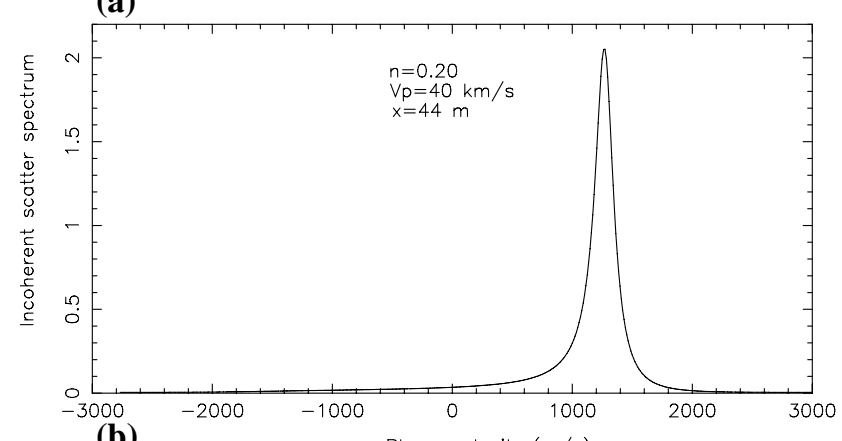

(b)

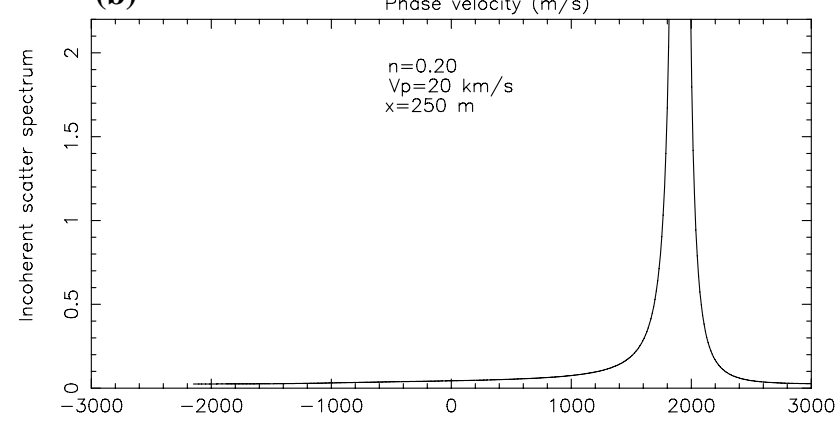

(c)

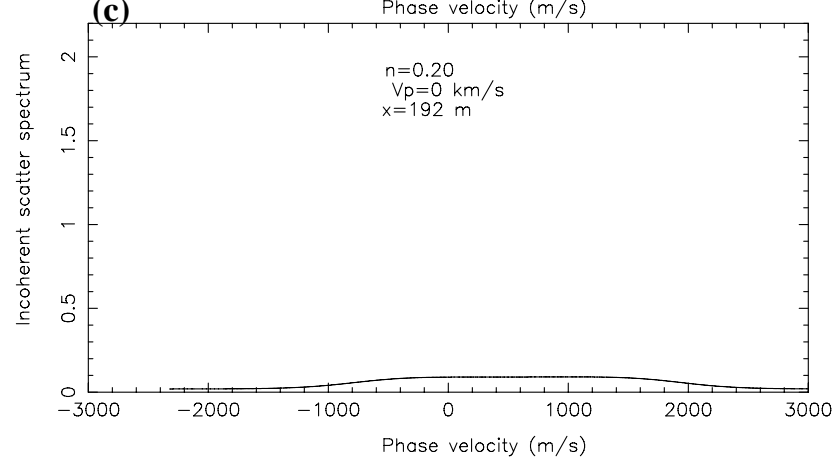

(d)

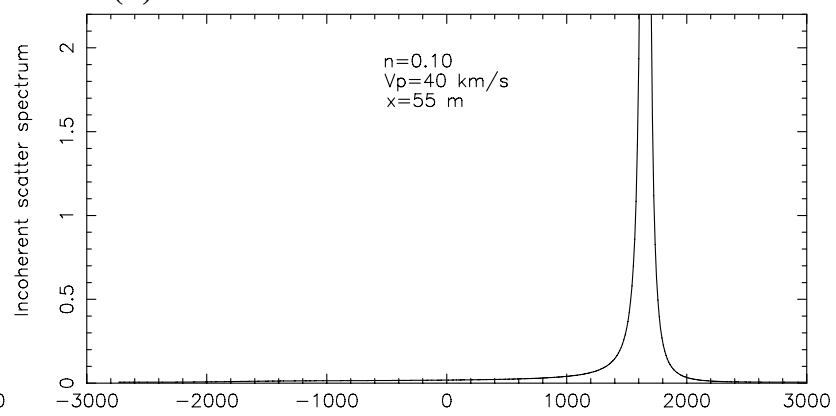

(e)

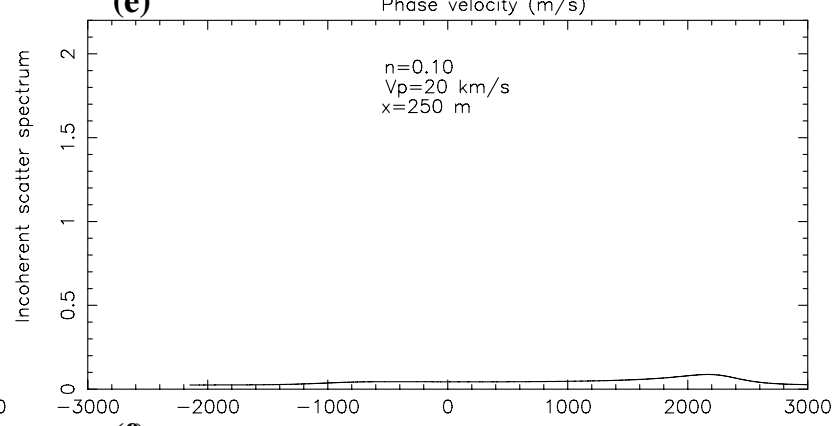

(f)

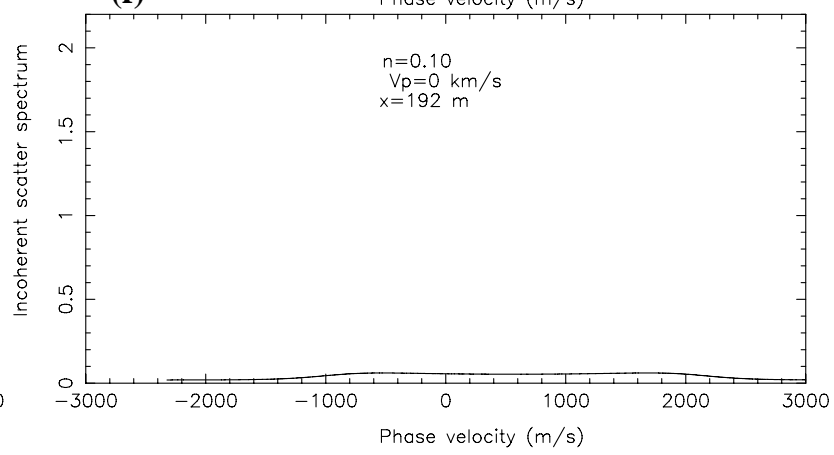

Fig. 12. The incoherent scatter spectra for the EDFs shown in Fig. 5. 
results here show otherwise. For a $10 \mathrm{~m}$ transverse wave scale, $V_{d e}^{\prime}$ will be on the order of $1000 \mathrm{~s}^{-1}$. Choosing $k_{z} / k_{y}=0.1$, we will find that for $\mathrm{O}^{+} V_{d e}^{\prime} /\left(\mu u \Omega_{i}\right) \approx 2 \times 10^{-5}$. Furthermore, the ions in response to wave fields are essentially immobile in the $B_{\|}$direction, that is, the ion drift is on the order $m_{i} / m_{e}$ times less than the electron drift. Then, $V_{d i}^{\prime} /\left(u \Omega_{i}\right) \ll 1$. Therefore, according to the simulation results here and ignoring non-Maxwellian features of the particle distributions, the transient gradient in the relative magnetic field aligned drift as driven by the EIC wave has a negligible effect on the amplitude of IA oscillations.

Additionally, the role of ion temperature anisotropy in the growth of shear-modified IA waves is to change the propagation direction or $k_{z} / k_{y}$, which is a critical term of Ganguli's generalized dispersion relation (Spangler et al., 2002; Teodorescu et al., 2003). As explained above, for a wide range of $k_{z} / k_{y}$ values, the shear effect as part of EIC wave oscillations will be insignificant, and therefore ion temperature anisotropy needs to be addressed independently from the shear effect.

Determination of the relative significance of EDF distortion effect to shear and temperature anisotropy effect in the presence of strong parallel inhomogeneous flows requires an advanced higher-dimensional numerical simulation code.

The connection between EIC waves and NEIALs is supported by their certain observational features. For instance, they appear to occur in the same environment, in association with field-aligned currents and soft electron precipitation. Moreover, Grydeland et al. (2004) made interferometric radar observations of filamented structures and showed that the echoes originate from very localized regions $(\approx 300 \mathrm{~m}$ scales) perpendicular to the magnetic field at $500 \mathrm{~km}$ altitude. The FAST structures that we have shown have spatial extents (not scale size) between 70 and $400 \mathrm{~m}$, in satisfactory agreement with Grydeland's measurements.

It remains to be determined if the EIC-driven IA waves occupy a sufficiently large volume or have sufficiently large amplitudes to cause ISR backscatter enhancements with backscatter power comparable to that of NEIALs. Although the IA enhancements could occur only at a certain phase region of the EIC wave, the EIC wave has a much faster phase velocity along B, so the IA waves can be excited everywhere, as long as they are kept alive by "EIC pumping". We will address this question with a $2 \mathrm{~d}-3 \mathrm{v}$ kinetic code in a future work.

Finally, EIC waves have been associated with transversely energizing ions through a gyroresonant motion, and incoherent scatter radars have often detected enhanced ion lines in the presence of ion upflows (Wahlund et al., 1992; Stromme, 1999). The same ion cyclotron waves that energize ion upflows could possibly be causing enhanced incoherent backscatter.

\section{Conclusions}

The large-amplitude EIC wave fields in the low-altitude auroral ionosphere can lead to non-Maxwellian electron distributions with slopes in the distribution function that are unstable to IA waves. The FAST satellite detected such wave fields in the altitude regions where enhanced incoherent scatter was recorded. On the basis of the computed particle distributions in response to such fields and the corresponding dispersion relation and incoherent scatter spectrum calculations, radar ion lines can be enhanced by such large-amplitude ion waves through an "ion cyclotron wave-driven ion acoustic instability".

This study will be followed by a higher dimensional code and including the ion dynamics to do a more complete simulation. We will also be looking for additional evidence of enhanced incoherent scatter as indicator of EIC turbulence in the auroral regions by looking into a larger dataset of FAST satellite measurements and determining where and when EIC turbulence occur, and comparing with the onset of enhanced incoherent scatter.

Acknowledgements. This work was supported by NSF grant no. ATM-0334122 to SRI International.

Topical Editor M. Pinnock thanks E. Mishin and another anonymous referee for their help in evaluating this paper.

\section{References}

Agrimson, E., D'Angelo, N., and Merlino, R.: Excitation of ionacoustic-like waves by subcritical currents in a plasma having equal electron and ion temperatures, Phys. Rev. Lett., 86(23), 5282-5285, 2001.

Bahcivan, H., Cosgrove, R., and Tsunoda, R.: Parallel electron streaming in the high-latitude $\mathrm{E}$ region and its effect on the incoherent scatter spectrum, J. Geophys. Res., 111(A7), A07 306, doi:10.1029/2005JA011595, 2006.

Bering, E. A.: The plasma wave environment of an auroral arc: electrostatic ion cyclotron waves in the diffuse aurora, J. Geophys. Res., 89, 1635-1649, 1984.

Bering, E. A., Kelley, M. C., and Mozer, F. S.: Observations of an intense field aligned thermal flow and associated intense narrow band electric field oscillations, J. Geophys. Res., 80, 4612-4620, 1975.

Bernstein, I. B., Greene, J. M., and Kruskal, M. D.: Exact nonlinear plasma oscillations, Phys. Rev., 108, 546-550, 1957.

Bhatnagar, P. L., Gross, E. P., and Krook, M.: Model for collision processes in gases I. Small amplitude processes in charged and neutral one-component systems, Phys. Rev., 94, 511-525, 1954.

Bonnell, J., Kintner, P., Wahlund, J. E., Lynch, K., and Arnoldy, R.: Interferometric determination of broadband ELF wave phase velocity within a region of transverse auroral acceleration, Geophys. Res. Lett., 23(23), 3297-3300, 1996.

Cabrit, B., Opgenoorth, H., and Kofnan, W.: Comparison between EISCAT UHF and VHF backscattering cross section, J. Geophys. Res., 101, 2369-2376, 1996. 
Cattell, C., Bergmann, R., Sigsbee, K., Carlson, C., Chaston, C., Ergun, R., McFadden, J., Mozer, F. S., Temerin, M., Strangeway, R., Elphic, R., Kistler, L., Moebius, E., Tang, L., and Pfaff, R.: The association of electrostatic ion cyclotron waves, ion and electron beams and field-aligned currents: FAST observations of an auroral zone crossing near midnight, Geophys. Res. Lett., 25(12), 2053-2056, 1998

Collis, P. N., Haggstrom, I., Kaila, K., and Rietveld, M. T.: EISCAT radar observations of enhanced incoherent scatter spectra: Their relation to red aurora and field-aligned currents, Geophys. Res. Lett., 18, 1031-1034, 1991.

Dougherty, J. P. and Farley, D. T.: A theory of incoherent scattering of radio waves by a plasma, Proc. Roy. Soc., A259, 79-99, 1960.

Ergun, R. E., Carlson, C. W., McFadden, J. P., Mozer, F. S., Delory, G. T., Peria, W., Chaston, C. C., Temerin, M., Elphic, R., Strangeway, R., Pfaff, R., Cattell, C. A., Klumpar, D., Shelley, E., Peterson, W., Moebius, E., and Kistler, L.: FAST satellite observations of electric field structures in the auroral zone, Geophys. Res. Lett., 25(12), 2025-2028, 1998.

Forme, F., Ogawa, Y., and Buchert, S. C.: Naturally enhanced ion acoustic fluctuations seen at different wavelengths, J. Geophys. Res., 106(A10), 21 503-21 515, 2001.

Foster, J. C., Pozo, C. D., Groves, K., and St-Maurice, J. P.: Radar observations of the onset of current-driven instabilities in the topside ionosphere, Geophys. Res. Lett., 15, 160-163, 1988.

Ganguli, G., Lee, Y. C., and Palmadesso, P. J.: Kinetic theory for electrostatic waves due to transverse velocity shears, Phys. Fluids, 31, 823-838, 1988.

Ganguli, G., Keskinen, M. J., Romero, H., Heelis, R., Moore, T., and Pollock, C.: Coupling of microprocesses and macroprocesses due to velocity shear: An application to the low-altitude ionosphere, J. Geophys. Res., 99(A5), 8873-8889, 1994.

Gavrishchaka, V. V., Ganguli, S. B., and Ganguli, G. I.: Origin of low-frequency oscillations in the ionosphere, Phys. Rev. Lett., 80(4), 728-731, 1998.

Gavrishchaka, V. V., Ganguli, G. I., Scales, W. A., Slinker, S. P., Chaston, C. C., McFadden, J. P., Ergun, R. E., and Carlson, C. W.: Multiscale coherent structures and broadband waves due to parallel inhomogeneous flows, Phys. Rev. Lett., 85(20), 42854288, 2000.

Grydeland, T., Blixt, E. M., Lovhaug, U. P., Hagfors, T., Hoz, C. L., and Trondsen, T. S.: Interferometric radar observations of filamented structures due to plasma instabilities and their relation to dynamic auroral rays, Ann. Geophys., 22, 1115-1132, 2004, http://www.ann-geophys.net/22/1115/2004/.

Holback, B., Jacksen, A., Ahlen, L., Jansons, S. E., Eriksson, A. I., Wahlund, J. E., Carozzi, T., and Bergman, J.: LINDA - the Astrid-2 Langmuir probe instrument, Ann. Geophys., 19, 601610, 2001, http://www.ann-geophys.net/19/601/2001/.

Kindel, J. M. and Kennel, C. F.: Topside current instabilities, J. Geophys. Res., 76, 3055-3078, 1971.

LaBelle, J. and Kintner, P. M.: The measurement of wavelength in space plasmas, Rev. Geophys., 27(4), 495-518, 1989.

LaBelle, J., Kintner, P. M., Yau, A. W., and Whalen, B. A.: Large amplitude wave packets observed in the ionosphere in association with transverse ion acceleration, J. Geophys. Res., 91, 71137118, 1989.

Louarn, P., Wahlund, J. E., Chust, T., de Feraudy, H., Roux, A.,
Holback, B., Dovner, P. O., Eriksson, A. I., and Holmgren, G.: Observations of kinetic Alfvén waves by the Freja satellite, Geophys. Res. Lett., 21, 1847-1850, 1994.

Mishin, E. V. and Fiala, V.: Radiation of whistlers by the ion-acoustic turbulence in the ionosphere, J. Geophys. Res., 100(A10), 19695-19700, 1995.

Mozer, F. S., Ergun, R., Temerin, M., Cattell, C., Dombeck, J., and Wygant, J.: New features of time domain electric-field structures in the auroral acceleration region, Phys. Rev. Lett., 79(7), 12811284, 1997.

Muschietti, L., Ergun, R. E., Roth, I., and Carlson, C. W.: Phasespace electron holes along magnetic field lines, Geophys. Res. Lett., 26, 1093-1096, 1999.

Newman, D. L., Goldman, M. V., Spector, M., and Perez, F.: Dynamics and instability of electron phase-space tubes, Phys. Rev. Lett., 86, 1239-1242, 2001.

Nicholson, D. R.: Introduction to Plasma Theory, Wiley, New York, 1983.

Ott, E. and Farley, D. T.: Microinstabilities and production of short wavelength irregularities in the auroral F region, J. Geophys. Res., 80(34), 4599-4602, 1975.

Palmadesso, P. J., Coffey, T. P., Ossakow, S. L., and Papadopoulos, K.: Topside ionosphere ion heating due to electrostatic ion cyclotron turbulence, Geophys. Res. Lett., 1, 105-108, 1974.

Paschmann, G., Haaland, S., and Treumann, R. T. (Eds.): Auroral Plasma Physics, Kluwer, 2003.

Kontar, E. P. and Pécseli, H. L.: Nonlinear wave interactions as a model for naturally enhanced ion acoustic lines in the ionosphere, Geophys. Res. Lett., 32, L05110, doi:10.1029/2004GL02218, 2005.

Rietveld, M. T., Collis, P. N., van Eyken, A. P., and Lvhaug, U. P.: Coherent echoes during EISCAT UHF Common Programmes, J. Atmos. Terr. Phys., 58, 161-174, 1996.

Rowland, H. L. and Palmadesso, P. J.: One-dimensional direct current resistivity due to strong turbulence, Phys. Fluids, 24(5), 832838, 1981.

Satyanarayana, P., Chaturvedi, P. K., Keskinen, M. J., Huba, J. D., and Ossakow, S. L.: Theory of the current-driven ion cyclotron instability in the bottomside ionosphere, J. Geophys. Res., 90, 2209-2218, 1985.

Sedgemore-Schulthess, F. and St.-Maurice, J. P.: Naturally enhanced ion-acoustic spectra and their interpretation, Surv. Geophys., 22, 55-92, 2001.

Spangler, R. S., Scime, E. E., and Ganguli, G. I.: Parallel inhomogeneous flow in a thermally anisotropic plasma: The electrostatic ion-acoustic branch, Phys. Plasmas, 9(6), 2526-2533, 2002.

St-Maurice, J. P., Noel, J. M., and Perron, P. J.: An assessment of how a combination of shears, field-aligned currents and collisions affect $\mathrm{F}$ region ionospheric instabilities, J. Plasma Phys., 73, 69-88, 2007.

Stasiewicz, K., Bellan, P., Chaston, C., Kletzing, C., Lysak, R., Maggs, J., Pokhotelov, O., Seyler, C., Shukla, P., Stenflo, L., Streltsoz, A., and Wahlund, J. E.: Small scale Alfvénic structure in the aurora, Space Sci. Rev., 92, 423-533, 2000.

Stromme, A.: Anomalous ion spectra and ion upflow observed with the EISCAT Svalbard Radar, Cand. Scient. Thesis, University of Tromso, Tromso, 1999.

Stromme, A., Belyey, V., Grydeland, T., Hoz, C. L., Lovhaug, U. P., and Isham, B.: Evidence of naturally occurring wave-wave in- 
teractions in the polar ionosphere and its relation to naturally enhanced ion acoustic lines, Geophys. Res. Lett., 32, L05 103, doi:10.1029/2004GL0202, 2005.

Teodorescu, C., Reynolds, E. W., and Koepke, M. E.: Experimental verification of the shear-modified ionacoustic instability, Phys. Rev. Lett., 88(18), 185003 , doi:10.1103/PhysRevLett.88.185003, 2002.

Teodorescu, C., Koepke, M. E., and Reynolds, E. W.: On the role of ion temperature anisotropy in the growth and propagation of shear-modified ion-acoustic waves, J. Geophys. Res., 108(A1), 1043, doi:10.1029/2002JA009395, 2003.

Tsunoda, R. T.: High latitude F region irregularities: a review and synthesis, Rev. Geophys., 26(4), 719-760, 1988.
Wahlund, J. E., Forme, F. R. E., Opgenoorth, H. J., Persson, M. A. L., Mishin, E. V., and Volokitin, A. S.: Scattering of electromagnetic waves from a plasma: enhanced ion acoustic fluctuations due to ion-ion two-stream instabilities, Geophys. Res. Lett., 19, 1919-1922, 1992.

Wahlund, J. E., Opgenoorth, H. J., Forme, F. R. E., Persson, M. A. L., Haggstrom, I., and Lilensten, J.: Electron energization in the topside auroral ionosphere: On the importance of ionacoustic turbulence, J. Atmos. Terr. Phys., 55, 623-645, 1993.

Wahlund, J. E., Eriksson, A. I., Holback, B., Boehm, M. H., Bonnell, J., Kintner, P. M., Seyler, C. E., Clemmons, J. H., Eliasson, L., Knudsen, D. J., Norqvist, P., and Zanetti, L. J.: Broadband ELF plasma emission during auroral energization 1. Slow ion acoustic waves, J. Geophys. Res., 103, 4343-4376, 1998. 